CONFORMAL GEOMETRY AND DYNAMICS

An Electronic Journal of the American Mathematical Society

Volume 3, Pages 50-66 (April 12, 1999)

S $1088-4173(99) 00036-3$

\title{
THURSTON BOUNDARY OF TEICHMÜLLER SPACES AND THE COMMENSURABILITY MODULAR GROUP
}

\author{
INDRANIL BISWAS, MAHAN MITRA, AND SUBHASHIS NAG ${ }^{1}$
}

\begin{abstract}
If $p: Y \rightarrow X$ is an unramified covering map between two compact oriented surfaces of genus at least two, then it is proved that the embedding map, corresponding to $p$, from the Teichmüller space $\mathcal{T}(X)$, for $X$, to $\mathcal{T}(Y)$ actually extends to an embedding between the Thurston compactification of the two Teichmüller spaces. Using this result, an inductive limit of Thurston compactified Teichmüller spaces has been constructed, where the index for the inductive limit runs over all possible finite unramified coverings of a fixed compact oriented surface of genus at least two. This inductive limit contains the inductive limit of Teichmüller spaces, constructed by I. Biswas, S. Nag and D. Sullivan, Determinant bundles, Quillen metrics and Mumford isomorphisms over the Universal Commensurability Teichmüller Space, Acta Mathematica, 176 (1996), 145-169, as a subset. The universal commensurability modular group, which was constructed in the above mentioned article, has a natural action on the inductive limit of Teichmüller spaces. It is proved here that this action of the universal commensurability modular group extends continuously to the inductive limit of Thurston compactified Teichmüller spaces.
\end{abstract}

\section{INTRODUCTION}

Let $p: Y \longrightarrow X$, be any finite unramified covering map between two arbitrary compact Riemann surfaces $X$ and $Y$. Both surfaces are assumed to have negative Euler characteristic. By pulling back complex structures (or hyperbolic metrics) on $X$, via $p$, one obtains an embedding,

$$
\mathcal{T}(p): \mathcal{T}(X) \longrightarrow \mathcal{T}(Y)
$$

of the Teichmüller space of $X$ into the Teichmüller space of $Y$. In fact, $\mathcal{T}(p)$ is a proper holomorphic embedding between these Teichmüller spaces, isometric with respect to the Teichmüller metrics (see [BNS], [BN1], [NS], [BN2], [N2]). In these papers the inductive system of Teichmüller spaces arising from these embeddings, as $p$ runs over all pointed finite unramified coverings of $X$, was studied. This inductive limit of Teichmüller spaces, which will be denoted by $\mathcal{T}_{\infty}(X)$, carries a natural action of the universal commensurability modular group, denoted by $M C_{\infty}(X)$.

In fact, $M C_{\infty}(X)$ acts faithfully through biholomorphic automorphisms on $\mathcal{T}_{\infty}(X)$, as well as on its completion, $\mathcal{T}\left(H_{\infty}(X)\right)$, the latter being the Teichmüller space for the universal hyperbolic solenoid $H_{\infty}(X)$ (see [BNS], [BN1] for the details). This modular group is universal in the sense that it does not depend on the genus of $X$. It will be important for us to recall that the new modular group,

Received by the editors April 27, 1998 and, in revised form, January 28, 1999.

1991 Mathematics Subject Classification. 32G15, 30F60, 57M10, 57M50.

${ }^{1}$ Subhashis Nag passed away on December 22, 1998. 
$M C_{\infty}(X)$, coincides with the group of all (orientation preserving) virtual automorphisms, Vaut $\left(\pi_{1}(X)\right)$, of the fundamental group $\pi_{1}(X)$. See the works cited.

Now, Thurston discovered (see, for instance, $[\mathrm{FLP}]$ ) an intrinsic compactification of the Teichmüller space:

$$
\mathcal{T}^{T}(X)=\mathcal{T}(X) \cup\{\text { Thurston's compactifying sphere }\}
$$

enjoying the property that the action, on $\mathcal{T}(X)$, of each element of the modular (= mapping class) group $M C G(X)$, extends continuously as homeomorphisms of $\mathcal{T}^{T}(X)$. The space $\mathcal{T}^{T}(X)$ is homeomorphic to the closed Euclidean ball of dimension $6 g-6$, and the compactifying boundary is a sphere $S^{6 g-7}$, when the genus of $X$ is $g$.

A natural question that arises is to investigate whether or not the direct limit construction of $\mathcal{T}_{\infty}(X)$, and the action thereon of $M C_{\infty}(X) \cong \operatorname{Vaut}\left(\pi_{1}(X)\right)$, can be carried out in the framework of the Thurston-compactified Teichmüller spaces. In this paper we answer these queries affirmatively.

Our first aim here is to demonstrate that, corresponding to any arbitrary finite covering $p$, there is an embedding:

$$
\mathcal{T}^{T}(p): \mathcal{T}^{T}(X) \longrightarrow \mathcal{T}^{T}(Y)
$$

extending continuously the embedding map $\mathcal{T}(p)$ of (1.1). Moreover, the association of the continuous map $\mathcal{T}^{T}(p)$ to the covering $p$ is a contravariant functor from the category of compact surfaces, with homotopy classes of unbranched covering maps as morphisms, to the category of Thurston compactified Teichmüller spaces and injective maps between them as morphisms. It is interesting that the extension map $\mathcal{T}^{T}(p)$ has remarkably simple and natural descriptions in the various (apparently disparate) models of the Thurston boundary. These are spelled out by us in Theorem 1 and its proof.

The functorial nature of the construction immediately implies that one can create the inductive limit of the Thurston-compactified Teichmüller spaces:

$$
\mathcal{T}_{\infty}^{T}(X)=\underline{\lim } \mathcal{T}^{T}(Y)
$$

as the index runs over the directed set of pointed covers of $X$. We may fix a universal cover $\widetilde{X} \longrightarrow X$. For each finite index subgroup $\Gamma$ of the Galois group $G$ of the universal cover, the quotient $\tilde{X} / \Gamma$ is a finite unramified cover of $X$. The set of finite index subgroups of $G$ are partially ordered by reverse inclusion, i.e., $\Gamma \geq \Gamma_{1}$ if and only if $\Gamma \subseteq \Gamma_{1}$. If we consider the inductive limit in (1.4) with the index set running over the set of finite index subgroups of $G$, then it is easy to see that the inductive limit coincides with $\mathcal{T}_{\infty}^{T}(X)$.

We will show that the direct limit of the Thurston boundaries is homeomorphic to the unit sphere $S^{\infty}$ in the direct sum $\mathbb{R}^{\infty}$, and this inductive limit inherits several natural structures, including a projectivized piecewise integrally linear (PIL for short) structure and a piecewise symplectic structure, from the corresponding structures on the finite dimensional Thurston compactifications.

Remark. Since the inductive limit of Teichmüller spaces is not even a locally compact space, one cannot hope to have a compactification by attaching a boundary. Therefore, our result that the inductive limit of Thurston boundaries exists, and that it attaches naturally to $\mathcal{T}_{\infty}(X)$ as an infinite dimensional boundary sphere, is the best possible situation to hope for in this context. 
Furthermore, and this is one of our chief points, $M C_{\infty}(X)$ will act by homeomorphisms on this direct limit space $\mathcal{T}_{\infty}^{T}(X)$. The naturality of the entire construction is borne out by our results that, as for the action of the finite genus modular groups on Thurston boundary, so also the universal commensurability modular group acts preserving the PIL and the symplectic structure that we shall exhibit on the direct limit of the Thurston boundaries.

The modular group $M C G(X)$ is known to act properly discontinuously on $\mathcal{T}(X)$. But the action of $M C G(X)$ on the Thurston boundary is topologically transitive or minimal, and even ergodic [M1]. Correspondingly we prove that $M C_{\infty}(X)$ acts on the direct limit of the Thurston boundary spheres in a minimal fashion. This result is connected to the Ehrenpreis conjecture.

\section{The Thurston Compactification of $\mathcal{T}(X)$}

Let $\mathcal{T}(X)=\mathcal{T}_{g}$ denote the Teichmüller space of the closed oriented smooth surface $X$, of genus $g$ with $g \geq 2$. We recall that the Teichmüller space $\mathcal{T}(X)$ is the space of all hyperbolic metrics (or conformal structures, or complex structures) on $X$ where two structures are identified if there is an isometry (respectively, conformal mapping, or biholomorphism) between them that is homotopic to the identity map of $X$. The space $\mathcal{T}(X)$ is a contractible complex manifold of complex dimension $(3 g-3)$.

Let $\operatorname{Diff}^{+}(X)$ denote the group consisting of all orientation preserving diffeomorphisms of $X$, and let $\operatorname{Diff}_{0}(X)$ denote its connected component containing the identity map. An alternative description of $\operatorname{Diff}_{0}(X)$ is that it consists of all diffeomorphisms homotopic to the identity map. The mapping class group of $X$, namely:

$$
M C G(X)=\operatorname{Diff}^{+}(X) / \operatorname{Diff}_{0}(X)
$$

acts naturally on $\mathcal{T}(X)$. This action is proper and discontinuous, and the quotient space coincides with the moduli space $\mathcal{M}_{g}$ of isomorphism classes of Riemann surfaces of genus $g$.

W. Thurston found a natural compactification of the Teichmüller space by attaching a sphere of dimension $6 g-7$ to $\mathcal{T}_{g}$. The compactification is intrinsic, in the sense that it actually does not depend on the choice of any reference hyperbolic metric or complex structure on $X$.

Let $\mathcal{T}^{T}(X)$ denote the compactified Teichmüller space with its Thurston boundary. For our work in this article, we will need to briefly recapitulate various ways of introducing the Thurston boundary.

Measured foliations and $\mathcal{T}^{T}(X)$. A measured foliation on a smooth surface is a foliation with finitely many singularities of prescribed type, and the foliation comes equipped with an invariant transverse measure, invariant with respect to the Bott partial connection along the foliation. Let $\mathcal{M F}(X)$ denote the space of measure equivalence classes of such measured foliations on $X$. We recall that measure equivalence is the weakest equivalence relation generated by the pullback operation on foliations by transverse measure preserving diffeomorphisms isotopic to the identity, together with the Whitehead operations on saddle connections that join singular points. The details can be found in [FLP]. The space $\mathcal{M F}(X)$ has a piecewise linear structure.

Let $\mathcal{S}$ denote the set of free homotopy (equivalently, isotopy) classes of simple closed homotopically non-trivial curves on $X$. If $X$ is equipped with a hyperbolic 
metric, then for each element in $\mathcal{S}$, there is a unique closed geodesic, for the hyperbolic metric, representing that element. Thus, given any hyperbolic metric on the surface $X$, we can assign a real number to each member of $\mathcal{S}$, namely the length of the corresponding geodesic. That procedure gives an embedding of the Teichmüller space into the space of positive real valued functions on $\mathcal{S}$ :

$$
\text { length : } \mathcal{T}(X) \longrightarrow \mathbb{R}_{+}^{\mathcal{S}}-0 .
$$

On the other hand, the space of non-trivial measured foliations also sits embedded in the same space of functions on $\mathcal{S}$, as follows. Given a measured foliation $(\mathcal{F}, \mu)$, and given any $[\gamma] \in \mathcal{S}$, one assigns to $[\gamma]$ the infimum of the transverse $\mu$-measures over all representatives of the class $[\gamma]$. In this way, both $\mathcal{T}(X)$ and $\mathcal{M F}(X)$ can be embedded in the space $\mathbb{R}_{+}^{\mathcal{S}}-0$. One passes to the projective space and defines the Thurston compactification, $\mathcal{T}^{T}(X)$, as the embedded image of the Teichmüller space union with the image of the projectivized measured foliations.

$$
\mathcal{T}^{T}(X)=\mathcal{T}(X) \bigcup \mathcal{P} \mathcal{M} \mathcal{F}(X)
$$

We refer to $[\mathrm{FLP}]$ for more details.

Measured geodesic laminations and $\mathcal{T}^{T}(X)$. Given a hyperbolic metric on $X$, a geodesic lamination, $\lambda$, is a smooth foliation of a closed subset of $X$ by hyperbolic geodesics as the leaves. A measured geodesic lamination is a geodesic lamination equipped with a transverse measure which is invariant under the Bott partial connection on the normal bundle along the foliation. In other words, one provides a measure on each closed arc transverse to the leaves of the lamination such that the measure is invariant under any homotopy of the arc that respects $\lambda$. The space of projectivized measured geodesic laminations, $\mathcal{P} \mathcal{M L}(X)$, also completes the Teichmüller space in a fashion equivalent to that described in the previous paragraph. In fact (see [FLP], [PH], or $[\mathrm{CB}]$ ) there is a natural way to pass between $\mathcal{M L}(X)$ and $\mathcal{M F}(X)$, which demonstrates that the boundaries of Teichmüller space determined from either method can be canonically identified, and we have

$$
\mathcal{T}^{T}(X)=\mathcal{T}(X) \bigcup \mathcal{P} \mathcal{M L}(X)
$$

Currents and $\mathcal{T}^{T}(X)$. We recall some of the basic notions from [Bo1]. Let the universal cover of the Riemann surface $X$ be denoted by $\tilde{X}$, which is conformally equivalent to the hyperbolic plane $\mathbb{H}^{2}$. Let $G(\widetilde{X})$ be the space of all (unoriented) geodesics in $\widetilde{X}$ equipped with the compact open topology. A geodesic current is a positive measure on $G(\widetilde{X})$ which is invariant under the action of $\pi_{1}(X)$. The space $\mathcal{C}(X)$ of geodesic currents is equipped with the weak ${ }^{\star}$ uniform structure coming from the family of semi-distances $d_{f}$ defined as

$$
d_{f}(\alpha, \beta):=\left|\int_{\alpha} f-\int_{\beta} f\right|
$$

where $\alpha, \beta \in \mathcal{C}(X)$ and $f$ ranges over all compactly supported real valued continuous functions on $G(\widetilde{X})$.

The space of simple closed curves on $X$ can be naturally embedded in $\mathcal{C}(X)$ by associating to a closed curve $c$ the probability measure supported on $c$. The geometric intersection number of simple closed curves easily extends to a continuous non-negative symmetric bilinear function $i: \mathcal{C}(X) \times \mathcal{C}(X) \longrightarrow \mathbb{R}^{+}$. 
The map $m \longrightarrow L_{m}$ assigning to each hyperbolic metric $m$ on $S$ its Liouville current $L_{m}$ (see [Bo1]) induces a proper topological embedding of the Teichmüller space

$$
L: \mathcal{T}(X) \longrightarrow \mathcal{C}(X) \text {. }
$$

This embedding is a homeomorphism onto its image.

A measured geodesic lamination on $X$ defines a geodesic current $\alpha$, whose selfintersection number, $i(\alpha, \alpha)$ is zero. In fact, $\mathcal{M L}(X)$ gets identified with such currents (see section 3 of [Bo1]). Consequently, the light cone comprising geodesic currents of self-intersection zero is homeomorphic to $\mathcal{M L}(X)$. Therefore, passing to projectivized geodesic currents, one obtains a compactification of the image of $\mathcal{T}(X)$ under $L$, by attaching the Thurston boundary - now modeled as the space of projectivized geodesic currents of self-intersection zero.

Harmonic maps and $\mathcal{T}^{T}(X)$. M. Wolf in [W] produced a $C^{\infty}$ diffeomorphism of $\mathcal{T}(X)$ onto the $6 g-6$ dimensional real vector space consisting of holomorphic quadratic differentials $Q(X)=H^{0}\left(X, K_{X}^{2}\right)$ on the Riemann surface $X$.

Let $\sigma$ denote the Poincaré metric on the Riemann surface $X$. Given any hyperbolic metric $\rho$ on the $C^{\infty}$ surface $X$, representing a point of $\mathcal{T}(X)$, consider the unique harmonic map $w:(X, \sigma) \longrightarrow(X, \rho)$, that is homotopic to the identity map of $X$. The map $w$ is actually a diffeomorphism. By associating to $\rho$ the $(2,0)$ part of the pullback of the metric $\rho$ by $w$, Wolf's diffeomorphic model of $\mathcal{T}(X)$ is obtained :

$$
\Phi: \mathcal{T}(X) \longrightarrow Q(X, \sigma)=Q(X) .
$$

We may compactify $\mathcal{T}(X)$ by attaching to each ray (or half line) through the origin in the real vector space $Q(X)$ an ideal point. Wolf proves that this compactification is the same as Thurston's compactification. This model of $\mathcal{T}^{T}(X)$ will be very useful for our work. Note that this model gives a ray structure to the Teichmüller space and its Thurston boundary once a base point in $\mathcal{T}(X)$ is fixed.

\section{Finite coverings and the Thurston Boundary}

Let $X=\Delta / G$ be obtained from the unit disc $\Delta$ by quotienting it with a torsionfree co-compact Fuchsian group $G \subset P S L(2, \mathbb{R})$. So the fundamental group $\pi_{1}(X)$ is isomorphic to $G$. Indeed, there is a natural isomorphism once we fix a point of $\Delta$. Let $p: Y \longrightarrow X$, be a finite unbranched covering space over $X$ of degree $d$. The covering map $p$ corresponds to the choice of a subgroup $H\left(\cong \pi_{1}(Y)\right.$ ), of index $d$, within the Fuchsian group $G$.

The Teichmüller spaces of $X$ and $Y$ are canonically identified with the Teichmüller spaces of the groups $G$ and $H$ respectively. The Teichmüller spaces of these Fuchsian groups appear embedded within the universal Teichmüller space $\mathcal{T}(\Delta)$ corresponding to the trivial Fuchsian group (see, for instance, [N1] for this basic material). The space $\mathcal{T}(\Delta)$ is a non-separable, infinite dimensional complex Banach manifold.

Thus the finite dimensional Teichmüller spaces $\mathcal{T}(X) \cong \mathcal{T}(G)$, and $\mathcal{T}(Y) \cong$ $\mathcal{T}(H)$, appear within $\mathcal{T}(\Delta)$ as properly embedded complex submanifolds. The inclusion of $H$ in $G$ induces a Teichmüller metric preserving proper holomorphic embedding of $\mathcal{T}(G)$ in $\mathcal{T}(H)$. This embedding will be denoted by $\mathcal{T}(p)$.

Our first aim is to establish the following theorem. 
Theorem 1. Given the degree d covering map $p: Y \longrightarrow X$ between closed oriented hyperbolic surfaces, there is a natural map between the corresponding Thurstoncompactified Teichmüller spaces. In fact, there exists, functorially associated to $p$, a continuous and injective map:

$$
\mathcal{T}^{T}(p): \mathcal{T}^{T}(X) \quad \longrightarrow \quad \mathcal{T}^{T}(Y)
$$

such that $\mathcal{T}^{T}(p)$ is the continuous extension of the holomorphic embedding $\mathcal{T}(p)$ : $\mathcal{T}(X) \longrightarrow \mathcal{T}(Y)$. The map $\mathcal{T}^{T}(p)$ restricted to the Thurston boundary sphere (the compactifying locus) of $\mathcal{T}^{T}(X)$ can be given the following equivalent descriptions:

(i) By the work of F. Bonahon [Bo1], [Bo2], the Thurston-compactified Teichmüller space is described in terms of the $G$-invariant geodesic currents on the unit disc. Then $\mathcal{T}^{T}(p)$ is defined by sending any $G$-invariant geodesic current on the universal covering disc $\Delta$ to the same current considered as an $H$-invariant object.

(ii) In the model of $M$. Wolf $[\mathrm{W}]$, the space $\mathcal{T}(X)$ is identified with the space of quadratic differentials $Q(X)$. Pullback of holomorphic quadratic differentials by the covering map $p$ defines a linear embedding of $Q(X)$ into $Q(Y)$, which preserves the ray structure. The map $\mathcal{T}^{T}(p)$ is defined by sending the ideal point for any ray in $Q(X)$ to the ideal point of the image ray in $Q(Y)$ by the above linear embedding.

(iii) By the work of Hubbard and Masur $[\mathrm{HM}]$, the Thurston boundary of $\mathcal{T}(X)$ can be identified as the space of projective rays in the linear space of quadratic differentials on the Riemann surface $X$, since each $\phi \in Q(X)$ gives rise to a measured foliation class on $X$. The map $\mathcal{T}^{T}(p)$ on the Thurston boundary points is again given by the pullback, via $p$, of holomorphic quadratic differentials on $X$.

(iv) The Thurston boundary may be represented as the space of projectivized measured geodesic laminations on the surface. The inverse image under $p$ of any measured geodesic lamination on $X$ produces a measured geodesic lamination on $Y$. The map obtained this way coincides with $\mathcal{T}^{T}(p)$.

The above descriptions demonstrate also that $\mathcal{T}^{T}(p)$ is injective, as was the map $\mathcal{T}(p)$ itself.

Proof of Theorem 1.

Proof of (i) [Geodesic currents]. The group $\pi_{1}(Y)$ sits as a subgroup of $\pi_{1}(X)$ via the monomorphism $p_{*}$ induced by $p$. Evidently, $G$-invariant currents allow a natural pullback via any covering. Indeed, a current invariant under the base group is, a fortiori, invariant under any of its subgroups. Thus there is a forgetful inclusion map at the level of currents that corresponds to pulling back a $\pi_{1}(X)$ invariant geodesic current (on the hyperbolic disc) to the very same current now considered as a $\pi_{1}(Y)$-invariant current.

The crucial observation is the following assertion regarding the current representing the pullback metric. If the hyperbolic metric $m \in \mathcal{T}(X)$ is represented in Bonahon's model by the $\pi_{1}(X)$-invariant geodesic current $L_{m}$, then the pulled back hyperbolic metric $p^{*} m$ on the covering surface $Y$ is represented in Bonahon's model of $\mathcal{T}(Y)$ by the same geodesic current $L_{m},-$ considered as a $\pi_{1}(Y)$-invariant current.

The above assertion is immediate from the definition of the Liouville current $L_{m}$ [Bo1, page 145].

It may be useful to point out the following interpretation of the assertion. Recall that currents live as measures on the space of all hyperbolic geodesics on the universal covering disc $\Delta$. Now, for the currents corresponding to the surface $X$, one 
is looking at the $\pi_{1}(X)$-invariant linear slice in the space of all geodesic currents on $\Delta$. The group $\pi_{1}(Y)$ being a subgroup of $\pi_{1}(X)$, a $\pi_{1}(X)$-invariant current may be regarded as a $\pi_{1}(Y)$-invariant current via the vector space inclusion homomorphism between the two corresponding strata. This defines the lifting of currents through a covering.

Now one traces through Bonahon's identification of the Thurston boundary within the space of geodesic currents, as described in Section 2. It becomes immediately clear that the above forgetful map on currents gives the continuous extension of the map $T(p)$ that we are seeking. The proof of the existence of $\mathcal{T}^{T}(p)$, and also the description (i) of it, is complete.

Injectivity of $\mathcal{T}^{T}(p)$ : It is clear from this description, as well as from each of the other descriptions, that the extension $\mathcal{T}^{T}(p)$ of $\mathcal{T}(p)$ remains an injection. $\diamond$

Proof of (ii) [Wolf model]. Denote the space of quadratic differentials $H^{0}\left(X, K_{X}^{2}\right)$ by $Q(X)$. In our situation we have an unramified covering $p: Y \longrightarrow X$ of Riemann surfaces. Tracing through the Wolf diffeomorphisms, we observe the fundamental fact that the induced mapping between Teichmüller spaces: $\mathcal{T}(p): \mathcal{T}(X) \longrightarrow \mathcal{T}(Y)$, in the Wolf models of $\mathcal{T}(X)$ and $\mathcal{T}(Y)$, is actually given just by pullback of holomorphic quadratic differentials by the map $p$ :

$$
\mathcal{T}(p) \equiv p^{*}: H^{0}\left(X, K_{X}^{2}\right) \cong \mathcal{T}(X) \longrightarrow H^{0}\left(Y, K_{Y}^{2}\right) \cong \mathcal{T}(Y) .
$$

Indeed, it is enough to observe that hyperbolic metrics as well as harmonic diffeomorphisms simply lift via the covering $p$. Consequently, the pullback, by $p$, of the quadratic differential on $X$ corresponding to a given point of the Teichmüller space $\mathcal{T}(X)$ coincides with the $(2,0)$ part of the pullback of the Kähler form on $Y$ by the harmonic diffeomorphism representing the point of $\mathcal{T}(Y)$ corresponding to the given point of $\mathcal{T}(X)$.

Since $p^{*}$ is a scalar multiple of an isometry (in the $L^{1}$ norm on quadratic differentials - the scalar being the degree of the covering), this description of Thurston compactification due to Wolf immediately implies that the embedding extends to the Thurston-compactifications, as desired. Indeed, $\mathcal{T}(p)$ is a linear map in this model of the Teichmüller spaces, and the ray structure is preserved. Thus (ii) of Theorem 1 is established also.

Proof of (iii) [Hubbard-Masur model]. We will now look at the Thurston boundary of $\mathcal{T}(X)$ as the projective classes of holomorphic quadratic differentials with respect to an arbitrarily assigned but fixed complex structure on $X$. We note that the main result of $[\mathrm{HM}]$ says that every measured foliation class in $\mathcal{M F}(X)$ is realized as the horizontal trajectory structure arising from a unique holomorphic quadratic differential on $X$.

Recall that a holomorphic quadratic differential is called Strebel (or JenkinsStrebel) if all the non-singular trajectories of its horizontal foliation are closed curves $([\mathrm{Str}])$. It is clear that the pullback of a Strebel differential by any finite holomorphic covering produces again a Strebel differential on the covering surface. Our strategy will be to demonstrate that the map $\mathcal{T}^{T}(p)$ has the desired description, as pullback via $p$, on the Strebel differentials. The density of Strebel differentials in $Q(X)$ will then suffice to complete the proof.

Let us trace through the identification between $\pi_{1}(X)$-invariant geodesic currents on $\Delta$ that live on the light-cone, and the holomorphic quadratic differentials on the Riemann surface $X$. The horizontal trajectories of the quadratic differential give rise to a measured foliation on $X$. As noted, that measured foliation corresponds 
to a geodesic lamination on $X$. Finally, the geodesic lamination will correspond to a certain $\pi_{1}(X)$-invariant geodesic current on $\Delta$ in the sense explained in Section 2 [Bo1, page 153]. That is how the three different descriptions of Thurston boundary: $\mathcal{T}^{T}(X)-\mathcal{T}(X)$, [namely: (1) measured foliations/quadratic differential trajectories, (2) measured geodesic laminations, (3) geodesic currents], get canonically identified with each other.

Consider now a Strebel differential, $q \in Q(X)$, with just one cylinder. That cylinder is swept out by the free homotopy class of some simple closed curve (called the core curve) $\gamma$, on $X$. (The height or modulus of that cylinder is not material to our present considerations.) The corresponding geodesic lamination on $X$ consists of just the unique hyperbolic geodesic in the free homotopy class of $\gamma$, with transverse measure being the Dirac measure on $\gamma$. But then the corresponding $\pi_{1}(X)$-invariant geodesic current is the Dirac measure supported on the union of all the hyperbolic geodesics in $\Delta$ that arise as the inverse image of $\gamma$ (in its geodesic position) by the universal covering projection from $\Delta$ onto $X$.

We have therefore identified the (light-cone) current corresponding to the Strebel point $q$. Now, by our already established description (i) of $\mathcal{T}^{T}(p)$ at the level of currents, we see that this current must map to the same current thought of as $\pi_{1}(Y)$ invariant current. But on $Y$ the pullback Strebel differential, $p^{*} q$, corresponds, by the same discussion as above, to exactly this $\pi_{1}(Y)$-invariant current. Consequently, $\mathcal{T}^{T}(p)$ has the description (iii) when acting on Strebel points of $Q(X)$.

Note. An alternative and instructive way to see the above is as follows. We know that $q$ will determine a point, say $b$, on the Thurston boundary of $\mathcal{T}(X)$. We claim that this point, $b$, is the limit in $\mathcal{T}^{T}(X)$ of a sequence of points, say $t_{n}$, of the Teichmüller space corresponding to pinching the curve $\gamma$. In fact, the hyperbolic length of the closed geodesic in the class of $\gamma$ is converging to zero as we go along the degenerating sequence of metrics. Hence the limit measured foliation on the Thurston boundary must have trajectory structure that assigns zero mass to the loop $\gamma$. So the loop $\gamma$ must not intersect transversely the leaves of the foliation $b$, or in other words, the leaves of $b$ must be parallel to $\gamma$. It is therefore easy to see that the point $b$ is given by the horizontal trajectory structure of the Strebel differential $q$. Now pull back the hyperbolic metrics $t_{n}$ to the corresponding sequence of hyperbolic metrics on the covering surface $\mathrm{Y}$. This lifted sequence in $\mathcal{T}(Y)$ will evidently converge to the boundary point of $\mathcal{T}^{T}(Y)$ represented by the pullback of the Strebel differential $q$. Thus $\mathcal{T}^{T}(p)$ is indeed defined on these Strebel boundary points by pullback of the relevant holomorphic quadratic differentials.

It now follows that $\mathcal{T}^{T}(p)$ in the entire quadratic differential picture must be pullback via $p$ on arbitrary (projective class of) quadratic differential, since this operation is continuous and coincides with the $\mathcal{T}^{T}(p)$ action on the dense set of Strebel points. That density, even for Strebel differentials with just one cylinder, is a result of Douady and Hubbard [DH]. This finishes the proof of part (iii) in the statement of the theorem.

Lastly, from the canonical identification : $\mathcal{M F}(X)=\mathcal{M L}(X)$, it follows that description (iv) of $\mathcal{T}^{T}(p)$, in terms of lifting laminations, is valid too.

This completes the proof of Theorem 1 .

Remark. Having established the existence of $\mathcal{T}^{T}(p)$, the association $p \longmapsto \mathcal{T}^{T}(p)$ can easily be seen to be a contravariant functor from the category of closed oriented 
surfaces, morphisms being homotopy classes of unbranched covering maps, to the category of Teichmüller spaces with Thurston boundary, and continuous injections thereof.

We will now work in the pointed category (for surfaces and covering maps); the factoring maps, whenever they exist, are therefore uniquely determined. Consequently, the compactified Teichmüller spaces $\mathcal{T}^{T}(Y)$, with the connecting maps $\mathcal{T}^{T}$ (.) between them, fit together into an inductive system, as desired.

We remark that it is possible to avoid the choice of a base point if we fix once and for all a universal cover $\widetilde{X}$ of $X$. In that situation, the coverings $X$ that will be considered are those which are a obtained from $\widetilde{X}$ by quotienting it with a finite index subgroup of the Galois group.

Definition 3.3. Denote by $\mathcal{T}_{\infty}^{T}(X)$ the direct limit of the $\mathcal{T}^{T}(Y)$ taken over the directed set of all pointed covers, $Y \longrightarrow X$, having range $X$.

As sketched in the introduction, instead of all possible covers of $X$ considered in the above definition, it is enough to consider a special of covers. We will describe it here in more details. Since $X$ is equipped with the choice of a base point $x \in X$, by considering homotopy classes of paths on $X$ starting at $x$, we get the universal cover

$$
\pi:(\tilde{X}, \tilde{x}) \longrightarrow(X, x)
$$

of the pointed surface $(X, x)$. Let $G$ denote the Galois group for the covering $\pi$, which is canonically isomorphic to the fundamental group $\pi_{1}(X, x)$. If $\Gamma \subset G$ is a subgroup of finite index, then $\widetilde{X} / \Gamma$ is a finite unramified pointed covering of $(X, x)$. The base point in $\widetilde{X} / \Gamma$ is the image of the point $\tilde{x}$. It is easy to see that any finite unramified pointed covering $Y \longrightarrow X$, where $Y$ is connected, is isomorphic to a covering of the above type for some $\Gamma \subseteq G$. Consequently, the direct limit of $\mathcal{T}^{T}(Y)$, where the index set runs over all pointed covers of $(X, x)$ given by subgroups $\Gamma \subset G$ of finite index, is canonically isomorphic to the direct limit $\mathcal{T}_{\infty}^{T}(X)$ in Definition 3.3 .

The final upshot is that one obtains a limit of spheres in Euclidean vector spaces namely a standard topological $S^{\infty}$ as the direct limit of the Thurston boundaries of the finite-dimensional Teichmüller spaces. So we have

$$
\mathcal{T}_{\infty}^{T}(X)-\mathcal{T}_{\infty}(X)=S^{\infty} .
$$

Pictured in the Wolf model, this limiting sphere $S^{\infty}$ can be thought of as the space of rays in the directed union of the vector spaces of holomorphic quadratic differentials, as one goes through the directed set of coverings over $X$.

Remark. C. Odden [Od] has taken some preliminary steps toward a theory of currents that live directly on the inverse limit solenoid $H_{\infty}(X)$. If that theory can be further worked out, it may be interesting to discover the relationship between the Thurston limit sphere, $S^{\infty}$, that we have found above, and some suitable projectivization of the space of solenoidal currents.

Action of the universal commensurability modular group. Recall from the work in [BNS] and [BN1], that the universal commensurability mapping class group,

$$
M C_{\infty}(X)=\operatorname{Vaut}\left(\pi_{1}(X)\right)
$$

acts faithfully on $\mathcal{T}_{\infty}(X)$ as biholomorphic automorphisms. 


\section{Theorem 2.}

1. Commensurability action: The action of the universal commensurability modular group $M C_{\infty}(X)=\operatorname{Vaut}\left(\pi_{1}(X)\right)$ extends, as self-homeomorphisms, on the inductive limit of the Thurston compactifications: namely on $\mathcal{T}_{\infty}^{T}(X)$.

2. Minimality at infinity: Every orbit of the above action of $M C_{\infty}(X)$, on the limit $S^{\infty}$ of the Thurston spheres, is dense in $S^{\infty}$.

Proof. For the first part of the theorem, the necessary set-theoretic idea follows the work in [BNS] and [BN1]. First, there is a natural map induced by the cover $p$, as follows:

$$
\mathcal{T}_{\infty}^{T}(p): \mathcal{T}_{\infty}^{T}(Y) \longrightarrow \mathcal{T}_{\infty}^{T}(X)
$$

In fact, $\mathcal{T}_{\infty}^{T}(p)$ is defined by mapping any point belonging to any Teichmüller space of a covering, say $Z$ over $Y$, to the same point of the same Teichmüller space, $\mathcal{T}(Z)$, where $Z$ is now considered as a covering over $X$ by composing the covering $Z \longrightarrow Y$ with $p$. It follows directly from the definition that $\mathcal{T}_{\infty}^{T}(p)$ is injective. Moreover, it is easily shown to be surjective by using a fiber-product argument on covering spaces. Thus each of these mappings, $\mathcal{T}_{\infty}^{T}(p)$, is an invertible homeomorphism between the universal Thurston-compactified commensurability Teichmüller spaces built from bases $Y$ and $X$ respectively. As a consequence, the group $M C_{\infty}(X)$, which was defined in [BNS] as the group arising from arbitrary cycles of covering arrows starting and ending at $X$, acts as automorphisms on $\mathcal{T}_{\infty}^{T}(X)$. Note that the association $p \longmapsto \mathcal{T}_{\infty}^{T}(p)$ is a covariant functor. This completes the proof of part (i) of the theorem.

The second part of the theorem arises from the fundamental fact ([FLP], [M2]) that, for each fixed surface $Y$, the modular group, $M C G(Y)$, acts with dense orbits on the Thurston sphere at the boundary of $\mathcal{T}(Y)$. The group $M C G(Y)$ is canonically isomorphic to the quotient by the group of inner automorphisms of $\pi_{1}(Y)$ of the subgroup Aut $\left(\pi_{1}(Y)\right)_{0}$ of the automorphism group Aut $\left(\pi_{1}(Y)\right)$ consisting of all those elements that act trivially on $H_{2}(Y, \mathbb{Z})=\mathbb{Z}$ (i.e., all those automorphisms of $\pi_{1}(Y)$ that arise from orientation preserving diffeomorphisms of $Y$ ). The group $\operatorname{Aut}\left(\pi_{1}(Y)\right)_{0}$ is contained in the universal commensurability modular group $M C_{\infty}(X)$. After identifying $M C_{\infty}(X)$ with $\operatorname{Vaut}\left(\pi_{1}(X)\right)$, the homomorphism of $\operatorname{Aut}\left(\pi_{1}(Y)\right)_{0}$ into $M C_{\infty}$ is the obvious one.

The topology of the sphere $S^{\infty}$ obtained by taking the inductive limit is merely the quotient topology from the disjoint union (co-product) topology of the individual strata. Moreover, the universal commensurability modular group contains faithful copies of the modular groups acting on the strata, (indeed these elements of $M C_{\infty}(X)$, which comprise a proper subset of $M C_{\infty}(X)$, were called the mapping class like elements; see [BN1], [Od]). Thus, it follows that it is sufficient to employ just the subset of mapping class like elements of $M C_{\infty}(X)$ alone, in order to show that each orbit of $M C_{\infty}(X)$ on the limiting sphere $S^{\infty}$ is dense.

Remark. In earlier papers, ([BNS], [BN1]), it was pointed out that the Ehrenpreis conjecture - regarding proximity of the complex structures on an arbitrary pair of compact Riemann surfaces with respect to taking finite unramified covers - is actually equivalent to the statement that the orbits of the action of $M C_{\infty}(X)$ on $\mathcal{T}_{\infty}(X)$ are dense. The result of Theorem $2(\mathrm{ii})$ above, that $M C_{\infty}(X)$ acts with dense orbits on the limit of Thurston boundaries, may be a bit of evidence for the validity of the Ehrenpreis conjecture. 
Remark. In [M1], Masur has shown that the action of $M C G(X)$ on the Thurston sphere $\mathcal{T}^{T}(X)-\mathcal{T}(X)$, is actually ergodic with respect to a suitable measure class. The theory of measures does not fit well with inductive limit constructions. On the other hand, if we consider a projective limit of measure spaces, the Kolmogorov existence theorem ensures the existence of a measure on the projective limit once the mappings are compatible with the measures. That is why a natural ergodicity statement for the action of the universal commensurability modular group on the limiting Thurston sphere $S^{\infty}$ is not possible.

Connected to this measure theoretic point, there is, however, an interesting matter that we wish to briefly indicate. It is possible to create a natural projective limit of the spaces of quadratic differentials on the covering surfaces. One takes the connecting maps in the inverse system to be the averaging map that sends quadratic differentials on $Y$ to those on $X$. Identifying the spaces of quadratic differentials, using the Wolf model, to the corresponding Teichmüller spaces, one thus does obtain an inverse limit of Teichmüller spaces. Indeed we can now show the existence of an inverse limit measure on the limit object, (a measure whose conditional expectations fit coherently).

This construction depends on the choice of a base complex structure on $X$, and it therefore transpires that $M C_{\infty}(X)$ does not have a natural action on the inverse limit. In fact, it is the commensurability automorphism group of the Riemann surface $X, \operatorname{ComAut}(X)$, (see [BN2], [BN3]) that acts on the inverse limit object. The group ComAut $(X)$ is actually the isotropy subgroup for the action of $M C_{\infty}(X)$ on $\mathcal{T}_{\infty}(X)$ at the point of $\mathcal{T}_{\infty}(X)$ represented by $X$. We refer to the papers cited above for the details.

The next section will be devoted to the construction of various natural structures on the inductive limit of the Thurston compactified Teichmüller spaces.

\section{PIL AND SYMPLECTIC STRUCTURES AT INFINITY}

For a given surface $X$, there exist finitely many train tracks carrying all laminations. This gives a coordinate chart system for the space of measured laminations $\mathcal{M L}(X)$. A diffeomorphism of the surface permutes these train tracks. Hence a diffeomorphism induces a piecewise integral map of $\mathcal{M L}(X)$. By considering the induced map of the projectivization of $\mathcal{M L}(X)$, one gets a piecewise integrally projective map of the Thurston boundary - which is a sphere with a PL structure coming from the train tracks. Now, lifting to covers preserves this structure - one needs to extend the basis given by train tracks at each stage. The outcome is that one obtains on the limiting infinite dimensional sphere, $S^{\infty}$, a natural PL structure. It is interesting to describe the train-track charts of this limiting sphere, and look at the action thereon of universal commensurability modular group $M C_{\infty}(X)$.

We note that the space $S^{\infty}$ is a $\mathbb{R}^{\infty}$-manifold [H1]. Consequently, $S^{\infty}$ is homeomorphic to $\mathbb{R}^{\infty}[\mathrm{H} 2$, page 48 , Corollary 2].

It is well-known that the space of measured laminations $\mathcal{M L}(X)$ on a surface $X$ of genus $g>1$ can be equipped with the following structures:

1. A PIL structure coming from charts corresponding to train tracks. (See, for instance, section 3.1 of $[\mathrm{PH}]$ ).

2. A piecewise bilinear skew-symmetric pairing coming from a family of such pairings, one corresponding to each train-track chart. (Section 3.2 of $[\mathrm{PH}]$.) 
Furthermore, any diffeomorphism $\phi$ of the surface preserves these structures, (vide addendum of $[\mathrm{PH}]$ on the action of the Mapping Class Group).

We will use terminology from the standard theory of train tracks on surfaces, as in $[\mathrm{PH}]$. Let us recall the notation:

$\mathrm{PIL}=$ Piecewise Integrally Linear,

$\mathrm{PIP}=$ Piecewise Integrally Projective.

These notions are meant to indicate the nature of the action of the mapping class group $M C G(X)$ of $X$ on measured lamination space, and projectivized measured lamination space, respectively.

We will briefly describe what PIL means (projectivizing one gets PIP).

(a) There exist finitely many train tracks $T_{1}, \cdots, T_{m}$ such that any lamination is carried by some $T_{i}$. (Note: all the $T_{i}$ may be chosen to have simply connected complements.)

(b) Each $T_{i}$ is regarded as a coordinate chart by associating to it all laminations carried by it. Furthermore, each $T_{i}$ gives a collection of equations whose solution is a cone on a polyhedron in Euclidean space.

Thus two such coordinate charts intersect along the laminations carried by two train tracks, corresponding to the situation when certain components of the solution-space are set to zero.

Hence given a choice of these finitely many $T_{i}$, the space $\mathcal{M L}(X)$ gets equipped with a piecewise linear structure - corresponding to the PL structure of a cone on a sphere. The sphere is equipped with a finite PL structure; each face of the sphere is a polyhedron again. As mentioned, the cone on a face can be regarded as the solution space to the equations given by the corresponding $T_{i}$.

Now look at the action of an element $\phi \in M C G(X)$ on the space $\mathcal{M L}(X)$ equipped with the above structure.

Claim 1: The action takes coordinate charts to coordinate charts. Indeed, this is a consequence of the fact that all the $T_{i}$ may be chosen to have simply connected complements.

Claim 2: Restrict to a coordinate chart. Recall that this means looking at all laminations carried by a particular track, $T_{1}$ say, and these are mapped to those carried by some $T_{2}$. (Alternately, one says $T_{1}$ is carried by $T_{2}$.) Now each branch of $T_{1}$ (the part of the track between 2 switches) is mapped to $T_{2}$ such that switches go to switches, hence the branch goes to an integral linear combination of strands of $T_{2}$. Thus the action on an integral solution is piecewise integral.

To see this even more explicitly, one can see that the image of a train track $\tau$ under a diffeomorphism is again a train track, which we will denote by $\sigma$. Further, this image train track is carried by one of the chosen train tracks. Now, using standard moves (alternately called peeling apart and its reverse pasting together) on train tracks, we can define a self-map of the surface homotopic to the identity such that $\sigma$ is mapped to one of the $T_{i}$ 's and switches go to switches.

Further a linear combination of solutions to equations coming from $T_{1}$ are sent to linear combinations of their images, i.e., the action is linear. The above condition is according to the law determined by strands going to integral linear combinations of strands. This is what one means by saying that the action of $\phi$ on $\mathcal{M L}(X)$ is PIL.

Projectivize the space $\mathcal{M L}(X)$. Then the resulting action of $\phi$ on $\mathcal{P} \mathcal{M L}(X)$ (this is the Thurston boundary), is called PIP. 
The PIL structure at infinity. There is an exact analog for the commensurability modular action in the direct limit situation we have been considering.

Let $G=\pi_{1}(X)$ and let

$$
H_{1}, H_{2}, \cdots, H_{i}, \cdots
$$

be an enumeration of the collection of all distinct subgroups of finite index in $G$. Let $X_{j} \longrightarrow X$ be the covering corresponding to the subgroup $H_{j}$. For each $X_{i}$ choose train tracks $T_{i 1}, \cdots, T_{i n_{i}}$ such that any lamination on $X_{i}$ is carried by some $T_{i j}$.

Let

$$
\mathcal{M L}_{\infty}(X)=\underset{\lim }{\longrightarrow} \mathcal{M L}\left(X_{i}\right)
$$

be the direct limit of the finite dimensional spaces of measured laminations as we run through all the finite coverings. Let $\lambda$ be an element of $\mathcal{M} \mathcal{L}_{\infty}(X)$. Then $\lambda$ is given by an equivalence class of some representative pair $\left(X_{i}, \lambda_{i}\right)$ where $\lambda_{i}$ is a measured lamination belonging to some $\mathcal{M L}\left(X_{i}\right)$.

Now, $\lambda_{i}$ is carried by one of the $T_{i j}$ 's by our choice of $T_{i j}$ 's. This shows that identifying $T_{i j}$ with the set of laminations carried by it, we have a countable collection of charts covering all of $\mathcal{M} \mathcal{L}_{\infty}(X)$. Furthermore, since train tracks lift to train tracks, the PIL structures fit together to give a PIL structure on $\mathcal{M} \mathcal{L}_{\infty}(X)$. Thus we have a PIL structure on $\mathcal{M} \mathcal{L}_{\infty}(X)$.

Piecewise bilinear skew-symmetric pairing. Let us now describe the piecewise bilinear skew symmetric pairing. It is shown in $[\mathrm{PH}]$ that for the subset $\mathcal{M L}(\tau) \subset$ $\mathcal{M L}(X)$ of laminations on the surface $X$ carried by the train track $\tau$ there exists a skew-symmetric bilinear pairing given as follows.

Let $w_{1}, w_{2}$ be two measured laminations, both carried by $\tau$. Then $w_{1}, w_{2}$ define 1 -cycles (also called $w_{1}, w_{2}$ for convenience) on $H_{1}(X)$. The fundamental (intersection) pairing for these laminations is given by

$$
\left(w_{1}, w_{2}\right)=\left(w_{1} \cup w_{2}\right) \cap[X] .
$$

These intersection pairing will fit together in $\mathcal{M} \mathcal{L}_{\infty}(X)$, provided we introduce a suitable normalizing factor. Thus, let $w_{i} \in \mathcal{M L}\left(X_{i}\right)$ and $w_{j} \in \mathcal{M L}\left(X_{j}\right)$ be two representative elements of $\mathcal{M} \mathcal{L}_{\infty}(X)$. Choose a common cover $X_{k}$ (the cover corresponding to $H_{i} \cap H_{j}=H_{k}$ is good enough) of $X_{i}, X_{j}$. Lift $w_{i}, w_{j}$ to measured laminations $u_{i}, u_{j}$ in $\mathcal{M L}\left(X_{k}\right)$. Finally define

$$
\left(w_{i}, w_{j}\right)_{\infty}=\frac{1}{g_{k}-1}\left(u_{i}, u_{j}\right)
$$

where the pairing between $u_{i}, u_{j}$ is the usual intersection pairing on the surface $X_{k}$ as defined in (4.3), and $g_{k}$ is the genus of $X_{k}$.

With this normalization, the pairings are easily seen to fit together to give a piecewise skew-symmetric bilinear pairing on $\mathcal{M L}_{\infty}(X)$. For the relevant computation, see the end of the proof of the next theorem.

We will consider the compatibility of the action of the universal commensurability modular group on the direct limit with the above structures. Let us note that the proof of Theorem 2(i) shows that the $M C_{\infty}(X)$ acts by homeomorphisms also on the (un-projectivized) space $\mathcal{M} \mathcal{L}_{\infty}(X)$.

Theorem 3. The direct limit $\mathcal{M L}_{\infty}(X)$ of measured laminations carries a natural piecewise integral linear structure equipped with a piecewise bilinear skew symmetric 
pairing. Further both these structures are preserved under the natural action of the universal commensurability modular group $M C_{\infty}(X)=\operatorname{Vaut}\left(\pi_{1}(X)\right)$. The action on the limit of Thurston boundaries, $S^{\infty}=\mathcal{P} \mathcal{M} \mathcal{L}_{\infty}(X)$, is therefore PIP.

Proof. In analogy with the situation in case of a single surface, we show that a virtual automorphism of $\pi_{1}(X)$ (which acts, by Theorem 2 , on $\mathcal{M} \mathcal{L}_{\infty}(X)$ ) preserves the structure described. Let $g$ be a virtual automorphism. One can choose a representative for $g$ as an isomorphism $g_{i j}: H_{i} \longrightarrow H_{j}$. That corresponds to a diffeomorphism between $X_{i}$ and $X_{j}$. (If $i=j$ we are in the situation of an automorphism of a surface - then the element $g$ is called mapping class like.)

As in the case of a single surface, $g_{i j}$ takes laminations carried by some $T_{i m}$ to laminations carried by some $T_{j n}$. Now $g_{i j}\left(T_{i m}\right)$ is clearly a train track on $X_{j}$. Furthermore, $g_{i j}\left(T_{i m}\right)$ is carried by $T_{j n}$, and can be mapped to $T_{j n}$ such that switches go to switches and branches are mapped to a sum of branches as in the case of a diffeomorphism of a single surface. (See above; the only new thing here is that we are considering a diffeomorphism between two possibly different surfaces.) This shows that the PIL structure is preserved by $g_{i j}$ since a branch goes to a positive integral linear combination of branches. In the direct limit one sees that $g$ preserves the PIL structure of $\mathcal{M} \mathcal{L}_{\infty}(X)$.

What we further desire to show is that $g$ preserves the piecewise skew-symmetric bilinear pairing of (4.4). To see this we use an equivalent description of members of $M C_{\infty}(X)$ by the 2-arrow diagrams that were introduced in [BNS], [BN1]. Then any $g \in M C_{\infty}(X)$ is given by two (in general inequivalent) covering maps, say $\pi_{1}, \pi_{2}$, from some surface $X_{i}$ onto the base surface, $X$.

Indeed, let $\mathcal{M L}_{\infty}\left(X_{i}\right)$ denote the directed system of spaces of measured laminations based at $X_{i}$. Induced by the covering $\pi_{1}$, just as in the proof of Theorem 2(i), we obtain a natural map:

$$
\mathcal{M L}_{\infty}\left(\pi_{1}\right): \mathcal{M L}_{\infty}\left(X_{i}\right) \longrightarrow \mathcal{M L}_{\infty}(X) .
$$

The action of the commensurability modular element, $g$, on $\mathcal{M} \mathcal{L}_{\infty}(X)$ is given by:

$$
\mathcal{M} \mathcal{L}_{\infty}\left(\pi_{2}\right) \circ \mathcal{M} \mathcal{L}_{\infty}\left(\pi_{1}\right)^{-1} .
$$

It is clearly enough to check that $\mathcal{M} \mathcal{L}_{\infty}\left(\pi_{1}\right)$ (and, similarly, $\mathcal{M} \mathcal{L}_{\infty}\left(\pi_{2}\right)$ ) preserves our intersection pairing.

This follows from the fact that we have normalized the intersection numbers on $X_{i}$ and $X$ by $\left(g_{i}-1\right)$ and $(g-1)$, respectively, and that the degree of the covering is precisely $\frac{g_{i}-1}{g-1}$. Indeed, let $u_{1}, u_{2}$ be laminations on $X$ pulling back by $\pi_{1}$, to $v_{1}$, $v_{2}$ on $X_{i}$. We show that the pairing is preserved:

$$
\begin{aligned}
\left(u_{1}, u_{2}\right)_{\infty} & =\frac{1}{g-1}\left(u_{1}, u_{2}\right) \\
\left(v_{1}, v_{2}\right)_{\infty} & =\frac{1}{g_{i}-1}\left(v_{1}, v_{2}\right) \\
& =\frac{1}{g_{i}-1}\left(v_{1} \cup v_{2}\right) \cap\left[X_{i}\right] \\
& =\frac{1}{g_{i}-1}\left(\left(u_{1} \cup u_{2}\right) \cap[X]\right) \frac{g_{i}-1}{g-1} \\
& =\left(u_{1}, u_{2}\right)_{\infty} .
\end{aligned}
$$

The proof of the theorem is finished. 


\section{InduCtive Limit of ThuRston-COMPACTIFIED MODUli SPACES}

The unramified finite covering $p: \widetilde{X} \longrightarrow X$ is called characteristic if it corresponds to a characteristic subgroup of the fundamental group $\pi_{1}(X)$. In other words, the subgroup $\pi_{1}(\tilde{X}) \subseteq \pi_{1}(X)$ must be invariant by every element of $\operatorname{Aut}\left(\pi_{1}(X)\right)$. This yields, therefore, a homomorphism :

$$
L_{p}: \operatorname{Aut}\left(\pi_{1}(X)\right) \longrightarrow \operatorname{Aut}\left(\pi_{1}(\widetilde{X})\right)
$$

The topological characterization of a characteristic cover is that every diffeomorphism of $X$ lifts to a diffeomorphism of $\widetilde{X}$, and the homomorphism (5.1) corresponds to this lifting process.

Characteristic subgroups are obviously normal. It is well-known that the normal subgroups of finite index form a co-final family among all subgroups of finite index in $\pi_{1}(X)$. This property continues to hold for the more special characteristic subgroups, as shown in [BN1].

Lemma ([BN1, Lemma 3.2]). The family of finite index characteristic subgroups, as a directed set partially ordered by inclusion, is co-final in the poset of all finite index subgroups of $\pi_{1}(X)$. In fact, given any finite covering $q: Y \longrightarrow X$, there exists another finite covering $h: Z \longrightarrow Y$ such that that the composition $q \circ h$ : $Z \longrightarrow X$ is a characteristic cover.

The characteristic tower. Consider the tower over the (pointed) surface $X=X_{g}$ consisting of only the characteristic coverings. Namely, we replace the old directed set, say $K(X)$ - consisting of all finite unramified pointed coverings, by the subset:

$$
K^{c h}(X) \subset K(X)
$$

consisting of all $\alpha$ such that $\alpha$ is a characteristic cover over $X$. Furthermore, for $\alpha, \beta$ in $K^{c h}(X)$, we say $\beta \succ \succ \alpha$ if and only if $\beta=\alpha \circ \theta$ with $\theta$ being also a characteristic covering. This gives $K^{\text {ch }}(X)$ the structure of a directed set.

As a consequence of the homomorphism (5.1), any characteristic cover $p$, from genus $\tilde{g}$ to genus $g$, induces a morphism

$$
\mathcal{M}(p): \mathcal{M}_{g} \longrightarrow \mathcal{M}_{\tilde{g}}
$$

which is an algebraic morphism between these normal quasi-projective varieties. In other words, the map $\mathcal{T}(p)$ descends to a map between the moduli spaces of Riemann surfaces when the covering $p$ is characteristic.

We therefore have a direct system of moduli spaces over the directed set $K^{\text {ch }}(X)$. Passing to the direct limit, we define:

$$
\mathcal{M}_{\infty}(X):=\lim _{\longrightarrow} \mathcal{M}\left(X_{\alpha}\right), \quad \alpha \in K^{c h}(X)
$$

in exact parallel with the definition of $\mathcal{T}_{\infty}(X)$.

We can now attach Thurston boundary at the moduli level. Define the Thurston compactification of each moduli space, as the quotient of $\mathcal{T}^{T}(X)$ by the corresponding modular group. The fact that the mapping class groups act ergodically and with dense orbits on the Thurston boundary spheres at each genus tells us that the quotient boundary is strongly non-Hausdorff. But nevertheless these compactified moduli spaces, with their weird boundaries, will fit together to give an inductive system of compactified moduli spaces. 
There is a natural subgroup Caut $\left(\pi_{1}(X)\right)$ as defined in [BN1], of the commensurability modular group, $M C_{\infty}(X)=\operatorname{Vaut}\left(\pi_{1}(X)\right)$, defined as the direct limit of the (base point preserving) modular groups as we go through the index set $K^{c h}(X)$.

Proposition 5.4. The subgroup Caut $\left(\pi_{1}(X)\right)$ of the commensurability modular group, acts on $\mathcal{T}_{\infty}^{T}(X)$ to produce the Thurston compactified ind-variety $\mathcal{M}_{\infty}^{T}(X)$ as the quotient.

Proof. Consider the direct system of Teichmüller spaces (with or without Thurston boundaries attached) over the co-final subset $K^{c h}(X)$. Let us denote by $\mathcal{T}_{\infty}^{c h}(X)$ the corresponding inductive limit space (without Thurston boundaries). But the inclusion of directed sets $K^{c h}(X)$ in $K(X)$ induces a natural homeomorphism of $\mathcal{T}_{\infty}^{c h}(X)$ onto $\mathcal{T}_{\infty}(X)$. It follows from the definition of the group Caut $\left(\pi_{1}(X)\right)$ that Caut $\left(\pi_{1}(X)\right)$ acts on $\mathcal{T}_{\infty}^{c h}(X)$ to produce $\mathcal{M}_{\infty}(X)$ as the quotient. Consequently, by identifying $\mathcal{T}_{\infty}^{c h}(X)$ with $\mathcal{T}_{\infty}(X)$ by the above homeomorphism, we obtain the result. Note that, because of set-theoretic generalities, the proof remains the same even when Thurston boundaries are attached all along.

\section{ACKNOWLEDGMENTS}

We are very grateful to the referee for going through the paper carefully and for many suggestions.

\section{REFERENCES}

[BNS] I. Biswas, S. Nag and D. Sullivan, Determinant bundles, Quillen metrics and Mumford isomorphisms over the Universal Commensurability Teichmüller Space, Acta Mathematica, 176, (1996), 145-169. MR 97h:32030

[BN1] I. Biswas and S. Nag, Weil-Petersson geometry and determinant bundles over inductive limits of moduli spaces, Lipa's Legacy (Ed. J.Dodziuk and L.Keen), 51-80, Contemporary Math, vol. 211, (1997), Amer. Math. Soc. CMP 98:03

[BN2] I. Biswas and S. Nag, Jacobians of Riemann surfaces and the Sobolev space $H^{1 / 2}$ on the circle, Mathematical Research Letters, 5, (1998), 281-292. CMP 98:16

[BN3] I. Biswas and S. Nag, Commensurability automorphism groups and infinite constructions in Teichmüller theory, Comptes Rendus Acad. Sci. (Paris), 327, (1998), 35-40.

[BN4] I. Biswas and S. Nag, Limit constructions over Riemann surfaces and their parameter spaces, and the commensurability group actions, Preprint 1998.

[Bo1] F. Bonahon, The geometry of Teichmüller space via geodesic currents, Inventiones Math, 92, 1988, 139-162. MR 90a:32025

[Bo2] F. Bonahon, Earthquakes on Riemann surfaces and on measured geodesic laminations, Trans. Amer. Math Soc., 330, 1992, 69-95. MR 92f:57021

[CB] A. Casson and S. Bleiler, Automorphisms of surfaces after Nielsen and Thurston, Cambridge Univ. Press, 1988. MR 89k:57025

[DH] A. Douady and J. Hubbard, On the density of Strebel differentials, Invent. Math., 30, (1975), 175-179. MR 53:796

[FLP] A. Fathi, F. Laudenbach and V. Poenaru, Travaux de Thurston sur le surfaces, Asterisque, vol 66-67, (1979).

[H1] R. E. Heisey, Embedding piecewise linear $\mathbb{R}^{\infty}$-manifolds into $\mathbb{R}^{\infty}$. The Proceedings of the 1981 Topology Conference (Blacksburg, Va., 1981), Topology Proc., 6, (1981), 317-328. MR 83k:57010

[H2] R. E. Heisey, Manifolds modelled on the direct limit of lines, Pacific Jour. Math., 102, (1982), 47-54. MR 84d:57009

[HM] J. Hubbard and H. Masur, Quadratic differentials and foliations, Acta Mathematica, 142, (1979), 221-274. MR 80h:30047

[M1] H. Masur, Interval exchange transformations and measured foliations, Annals of Math., 115, (1982), 169-200. MR 83e:28012 
[M2] H. Masur, Measured foliations and handlebodies, Ergodic Th. Dynam. Systems, 6, (1986), 99-116. MR 87i:57011

[N1] S. Nag, The Complex Analytic Theory of Teichmüller Spaces, Wiley-Interscience, New York, (1988). MR 89f:32040

[N2] S. Nag, Mathematics in and out of string theory, Proc. 37th Taniguchi Symposium "Topology and Teichmüller Spaces", Finland 1995, (Ed. S. Kojima, et. al.), World Scientific, (1996). CMP 99:06

[NS] S. Nag and D. Sullivan, Teichmüller theory and the universal period mapping via quantum calculus and the $H^{1 / 2}$ space on the circle, Osaka J. Math., 32, (1995), 1-34. MR 96c:32023

[Od] C. Odden, The virtual automorphism group of the fundamental group of a closed surface, Thesis, Duke University, 1997.

[PH] R. Penner and J. Harer, Combinatorics of train tracks, Annals Math Studies, Princeton Univ. Press, 1992. MR 94b:57018

[Str] K. Strebel, Quadratic differentials, Springer Verlag, Berlin. MR 86a:30072

[W] M. Wolf, The Teichmüller theory of harmonic maps, Jour. Diff. Geom., 29, 1989, 449-479. MR 90h:58023

School of Mathematics, Tata Institute of Fundamental Research, Homi Bhabha RoAD, Bombay 400005, India

E-mail address: indranil@math.tifr.res.in 